\title{
EXISTENCE, UNIQUENESS, AND ANGLE COMPUTATION FOR THE LOXODROME ON AN ELLIPSOID OF REVOLUTION
}

\author{
ABED ELHASHASH
}

Communicated by Gregory L. Naber

\begin{abstract}
We summarize a proof for the existence and uniqueness of the loxodrome joining two distinct points $p_{o}$ and $p_{1}$ on an open half of an ellipsoid of revolution. We also compute the unique angle $\alpha \in[0,2 \pi)$ which the loxodrome makes with the meridians intersecting the loxodrome.
\end{abstract}

\section{Introduction}

A loxodrome on an ellipsoid of revolution is a curve that traverses all the meridians along its way at a constant angle. Since the earth is modeled as an ellipsoid of revolution, understanding loxodromes plays an important role in the science of navigation; see, e.g., [4-6,9]. The existence and uniqueness of a loxodrome on an ellipsoid of revolution and a formula for its angle are known results; see, e.g., $[4,9]$.

Typically, the existence of a loxodrome on an ellipsoid of revolution is proved by constructing a one-to-one conformal map (is a continuously differentiable map that preserves angles between curves) $\Psi$ from the open square $\left(-\frac{\pi}{2}, \frac{\pi}{2}\right) \times\left(-\frac{\pi}{2}, \frac{\pi}{2}\right)$ $\subset \mathbb{R}^{2}$ onto an open connected subset of the ellipsoid of revolution which contains the points $p_{o}$ and $p_{1}$ that are meant to be joined by a loxodrome. The map $\Psi$ is such that every vertical straight line segment in the open square $\left(-\frac{\pi}{2}, \frac{\pi}{2}\right) \times\left(-\frac{\pi}{2}, \frac{\pi}{2}\right)$ is mapped onto a meridian of the ellipsoid of revolution. Thus, if $q_{o}=\Psi^{-1}\left(p_{o}\right)$ and $q_{1}=\Psi^{-1}\left(p_{1}\right)$, then $q_{o}$ and $q_{1}$ can be joined by a straight line segment in the open square $\left(-\frac{\pi}{2}, \frac{\pi}{2}\right) \times\left(-\frac{\pi}{2}, \frac{\pi}{2}\right)$ (which makes a constant angle with all vertical straight line segments on its way) and the image of that straight line segment joining $q_{o}$ to $q_{1}$ under $\Psi$ will be a curve on the ellipsoid of revolution joining $p_{o}$ to $p_{1}$ which makes a constant angle with all meridians on its way (because $\Psi$ is conformal). Moreover, the constant angle that the loxodrome makes with all the meridians is typically computed by using the technique of "infinitesimals"; see, e.g., [5, 6]. 
Our main aim in this paper is to present a rigorous mathematical proof that avoids the use of conformal maps for the existence and uniqueness of a loxodrome joining two distinct points $p_{o}$ and $p_{1}$ that lie on an ellipsoid of revolution and that differ by less than $\pi$ radians (modulo $2 \pi$ ) in longitude and to obtain a formula for the constant angle that the loxodrome makes with the meridians without the use of "infinitesimals".

In Section 2, we define the ellipsoid of revolution $\mathcal{E} \subset \mathbb{R}^{3}$ to be considered throughout this paper and we introduce some definitions and notation that we use in later sections. Then, in Section 3, we parameterize $\mathcal{E}^{+}:=\mathcal{E} \cap\{(x, y, z) \in$ $\left.\mathbb{R}^{3} ; x>0\right\}$, an open half of the ellipsoid $\mathcal{E}$, by latitude and longitude using a diffeomorphism $\psi$. After that, we use the diffeomorphism $\psi$ to parameterize unit tangent vectors pointing towards the north and the east, respectively, at any point on $\mathcal{E}^{+}$in terms of the longitude and latitude of that given point. We use the latter parametrization to formulate the problem of finding a loxodrome joining two distinct points in $\mathcal{E}^{+}$and we solve the problem in Section 4.

\section{Definitions and Notation}

Let $a$ and $b$ be real scalars such that $a \geq b>0$ and consider the set $\mathcal{E}:=$ $\left\{(x, y, z) \in \mathbb{R}^{3} ; \frac{x^{2}+y^{2}}{a^{2}}+\frac{z^{2}}{b^{2}}=1\right\}$. Then, $\mathcal{E}$ is an ellipsoid of revolution. Any point $p=(x, y, z) \in \mathcal{E}$ other than $(0,0, b)$ and $(0,0,-b)$ can be located by two real numbers: $\lambda$ in the interval $(-\pi, \pi]$ and $\phi$ in the open interval $\left(-\frac{\pi}{2}, \frac{\pi}{2}\right)$ where $\lambda=\lambda(p)$ is the longitude of $p \in \mathcal{E}$, i.e., the counter-clockwise angle measured in the $x y$-plane from the positive $x$-axis to the orthogonal projection (onto the $x y$-plane) of the straight line segment joining the origin to $p$, and $\phi=\phi(p)$ is the latitude of a point $p \in \mathcal{E}$, i.e., the angle (of elevation) that the normal line to $\mathcal{E}$ through $p$ makes with the $x y$-plane; refer to Fig. 1 for illustrations. In particular, if the point $p=(x, y, z) \in \mathcal{E}$ satisfies $x>0$ then the longitude $\lambda=\lambda(p)$ can be chosen in the open interval $\left(-\frac{\pi}{2}, \frac{\pi}{2}\right)$. Moreover, we note that for such a point $p=(x, y, z) \in \mathcal{E}$ having $x>0$ the longitude is positive if and only if $y>0$ and that the latitude is positive if and only if $z>0$. Define $\mathcal{E}^{+}:=\{(x, y, z) \in \mathcal{E} ; x>0\}$. By a meridian in $\mathcal{E}^{+}$, we mean a curve in $\mathcal{E}^{+}$ whose points have the same longitude and which is oriented in such a way that it starts at $(0,0,-b)$ and ends at $(0,0, b)$. Any curve in $\mathcal{E}$ obtained by rotating a meridian in $\mathcal{E}^{+}$around the $z$-axis shall also be called a meridian. By a semi-circle of latitude in $\mathcal{E}^{+}$, we mean a curve in $\mathcal{E}^{+}$whose points have the same latitude and which is oriented in a way that it starts at the point with the negative $y$-component and ends at the point with the positive $y$-component. If $p$ is a point in $\mathcal{E}$ other than 

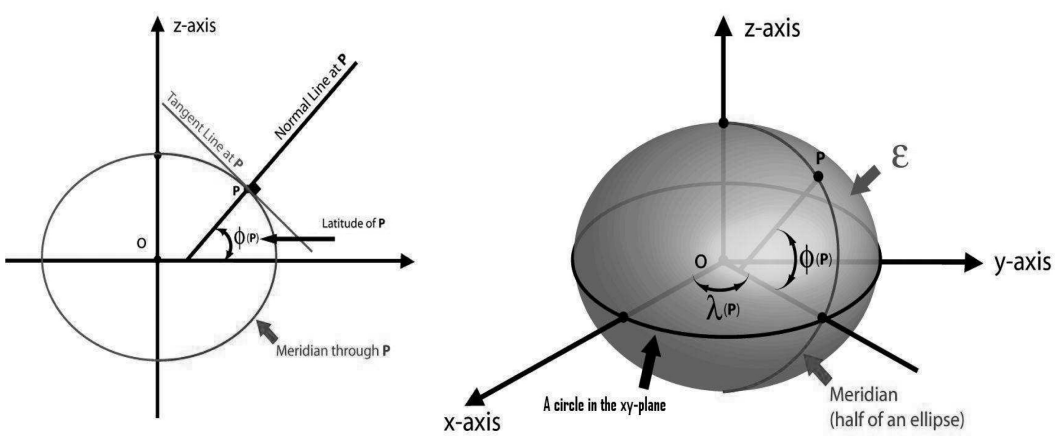

Figure 1. The longitude and latitude of a point $p$.

$(0,0, b)$ and $(0,0,-b)$ then we say that a non-zero tangent vector to the meridian at $p$ points towards the north if its $z$-component is positive. If $p$ is a point in $\mathcal{E}^{+}$ then we say that a non-zero tangent vector to the semi-circle of latitude at $p$ points towards the east if its $y$-component is positive. A loxodrome in $\mathcal{E}$ is a curve that intersects all the meridians on its way at a constant angle.

Furthermore, the vectors that we mention in this paper are all real two-dimensional or three-dimensional column vectors, i.e., 3-by-1 or 2-by-1 matrices with real entries. Moreover, we will enclose the entries of a vector by a pair of brackets to distinguish it from a point. The superscript ${ }^{T}$ will denote the transpose of a vector (treated as a matrix). For example, the vector $\mathbf{u}=\left[\begin{array}{lll}4 & 5 & 6\end{array}\right]^{T}$ is the transpose of the 1-by-3 matrix [ $\left.\begin{array}{lll}4 & 5 & 6\end{array}\right]$ and hence it is the 3-by-1 matrix whose (1,1)-entry is 4 , $(2,1)$-entry is 5 , and $(3,1)$-entry is 6.

If $\mathcal{M}, \mathcal{N} \subseteq \mathbb{R}^{3}$ are real submanifolds of $\mathbb{R}^{3}$ (see, e.g., [7,8]), $p$ is a point in $\mathcal{M}$, and $\psi: \mathcal{M} \rightarrow \mathcal{N}$ is a diffeomorphism (see, e.g., [7,8]), then $\left.\mathrm{d} \psi\right|_{p}$ will denote the derivative of $\psi$ at the point $p$. If it is clear at which point the derivative is being taken then we just write $\mathrm{d} \psi$.

Remark 1. We try to use the standard notation used by other authors in this subject. For instance, most of our notation is consistent with Pearson's notation [5]. Moreover, throughout this paper, we use the Greek letter $\lambda$ (respectively, the Greek letter $\phi$ ) sometimes to denote the longitude variable (respectively, the latitude variable) and some other times to denote a parametrization of that variable. To avoid confusion, the following convention is adopted: if the Greek letter $\lambda$ (respectively, the Greek letter $\phi$ ) is followed by a pair of parentheses enclosing a variable in $\mathbb{R}$, then it denotes the longitude variable (respectively, latitude vari- 
able) parameterized by the given real variable and if it is not followed by a pair of parentheses enclosing a variable then it simply denotes the longitude variable (respectively, the latitude variable).

For example, if $t \in \mathbb{R}$ then we have the following.

$$
\begin{aligned}
\phi & =\text { the latitude variable. } \\
\lambda & =\text { the longitude variable. } \\
\phi(t) & =\text { the latitude variable parametrized by a real variable } t . \\
\lambda(t) & =\text { the longitude variable parametrized by a real variable } t . \\
\lambda(\phi(t)) & =\text { the longitude variable parametrized by } \phi(t) . \\
\lambda(\phi) & =\text { the longitude variable parametrized by the latitude } \phi .
\end{aligned}
$$

\section{Parameterizing $\mathcal{E}^{+}$and Stating the Problem}

Since $\mathcal{E}$ is invariant under rotations around the $z$-axis and since we are interested in proving the existence and uniqueness of a loxodrome joining two distinct points whose difference in longitude is less than $\pi$ radians (modulo $2 \pi$ ), it is enough to prove the existence and uniqueness of a loxodrome joining two distinct points in the subset $\mathcal{E}^{+}$of the ellipsoid $\mathcal{E}$. To that end, we will do the following:

- we will parameterize $\mathcal{E}^{+}$in terms of latitude and longitude,

- we will express the unit tangent vectors pointing towards the north and the east at a point on some curve $\eta$ in $\mathcal{E}^{+}$in terms of the latitude, the longitude, and the parameter of the curve $\eta$, and

- we will state the problem of finding the loxodrome in $\mathcal{E}^{+}$.

In the following lemma, we give a parametrization of $\mathcal{E}^{+}$in terms of latitude and longitude. We postpone the proof of this lemma to the Appendix and we note that the parametrization presented in this lemma can be obtained from [5] or [9]. The details that we mention about the construction of this parametrization are presented for the sake of completeness.

Lemma 2. Let $J$ denote the interval $\left(-\frac{\pi}{2}, \frac{\pi}{2}\right)$ and let $e=\left(\left(a^{2}-b^{2}\right) / a^{2}\right)^{1 / 2}$ be the eccentricity of the ellipsoid $\mathcal{E}$. If the map $\psi: J \times J \rightarrow \mathcal{E}^{+}$is defined by

$$
\psi(\phi, \lambda)=\left(R(\phi) \cos \lambda, R(\phi) \sin \lambda, R(\phi)\left(1-e^{2}\right) \tan \phi\right)
$$


where

$$
R(\phi)=a \cos \phi\left(1-e^{2} \sin ^{2} \phi\right)^{-1 / 2}
$$

then the map $\psi$ is a diffeomorphism and its inverse diffeomorphism $\psi^{-1}: \mathcal{E}^{+} \rightarrow J \times J$ is given by:

$$
\psi^{-1}(x, y, z)=\left(\arctan \left(\frac{z}{\left(1-e^{2}\right) \sqrt{x^{2}+y^{2}}}\right), \arcsin \left(\frac{y}{\sqrt{x^{2}+y^{2}}}\right)\right) .
$$

Moreover, the first entry (respectively, the second entry) of $\psi^{-1}(x, y, z)$ is the latitude (respectively, the longitude) of the point $(x, y, z)$.

The following lemma gives explicitly the entries of the unit tangent vector pointing towards the north (respectively, the east) at a point on some curve in $\mathcal{E}^{+}$in terms of the latitude, the longitude, and the parameter of the curve. The proof of this lemma is postponed to the Appendix.

Lemma 3. Let $I$ denote a non-empty open interval and suppose that $\eta: I \rightarrow \mathcal{E}^{+}$is a curve and that $\gamma$ is the image of the curve $\eta$ under the diffeomorphism $\psi^{-1}$ defined in equation (3). If $\mathbf{N}_{\mathbf{t}}$ (respectively, $\mathbf{E}_{\mathbf{t}}$ ) denotes the three-dimensional unit vector at $\eta(t)$ that depends smoothly on $t$ and that points towards the north (respectively, the east), and $\gamma(t)$ is given by $\gamma(t)=(\phi(t), \lambda(t))$ for all $t \in I$, then the following statements are true

- $\mathbf{N}_{\mathbf{t}}=\left[\begin{array}{lll}-\sin \phi(t) \cos \lambda(t) & -\sin \phi(t) \sin \lambda(t) & \cos \phi(t)\end{array}\right]^{T}$ and

- $\mathbf{E}_{\mathbf{t}}=\left[\begin{array}{lll}-\sin \lambda(t) & \cos \lambda(t) & 0\end{array}\right]^{T}$.

Moreover, if

$$
r(\phi):=a\left(1-e^{2}\right)\left(1-e^{2} \sin ^{2} \phi\right)^{-3 / 2}
$$

$R(\phi)$ is given by equation (2), and $\mathbf{n}_{\mathbf{t}}$ (respectively, $\mathbf{e}_{\mathbf{t}}$ ) denotes the two-dimensional vector which is the image of $\mathbf{N}_{\mathbf{t}}$ (respectively, $\mathbf{E}_{\mathbf{t}}$ ) under $\left.\mathrm{d} \psi^{-1}\right|_{\eta(t)}$, then we have

$$
\begin{aligned}
& \text { - } \mathbf{n}_{\mathbf{t}}=\left[\begin{array}{ll}
\frac{1}{r(\phi(t))} & 0
\end{array}\right]^{T} \text { and } \\
& \text { - } \mathbf{e}_{\mathbf{t}}=\left[\begin{array}{ll}
0 & \frac{1}{R(\phi(t))}
\end{array}\right]^{T} \text {. }
\end{aligned}
$$

Remark 4. It is useful to keep in one's mind as one reads this article that the eccentricity $e$ of the ellipsoid $\mathcal{E}$ is a real number in the interval $[0,1)$ and that 
$R(\phi)$ (respectively, $r(\phi))$ as defined in equation (2) (respectively, equation (4)) is positive for all $\phi$ in the open interval $\left(-\frac{\pi}{2}, \frac{\pi}{2}\right)$. Moreover, we mention in passing that $R(\phi)$ is the radius of the semi-circle of latitude at a latitude equal to $\phi$ and that $r(\phi)$ is the radius of curvature of the meridian at any point whose latitude is $\phi$; see, e.g., [5].

In what follows, if a function of one variable is followed by the superscript ' (prime) then this denotes the derivative with respect to the real variable $t$.

The problem of finding a loxodrome can be stated using the notation of Lemma 3 as follows:

Problem 1. Given two distinct points $p_{o}$ and $p_{1}$ in $\mathcal{E}^{+}$, we want to find a curve $\eta$ in $\mathcal{E}^{+}$defined on an open interval I that contains 0 and a real number $\alpha$ in the interval $[0,2 \pi)$ such that:

- $\eta(0)=p_{o}, \eta(\epsilon)=p_{1}$ for some positive scalar $\epsilon \in I$, and

- $\eta^{\prime}(t)=\cos \alpha \mathbf{N}_{\mathbf{t}}+\sin \alpha \mathbf{E}_{\mathbf{t}}$ for all $t$ in the closed interval $[0, \epsilon]$.

\section{Joining the Points}

In this section, we will solve Problem 1. Moreover, we mention here that from this point onward we shall use the notation of Lemma 2, Lemma 3, and Problem 1. So, $J$ will denote the open interval $\left(-\frac{\pi}{2}, \frac{\pi}{2}\right) ; e$ will denote the eccentricity of the ellipsoid $\mathcal{E}$ as given in Lemma 2; $\psi$ is given by equation (1); $r(\phi)$ is given by equation (4); $R(\phi)$ is given by equation (2); and the tangent vectors $\mathbf{N}_{\mathbf{t}}, \mathbf{E}_{\mathbf{t}}, \mathbf{n}_{\mathbf{t}}$, and $\mathbf{e}_{\mathbf{t}}$ are as given in Lemma 3 . We begin by considering the following problem:

Problem 2. Let I be an open interval that contains 0 and let $\left(\phi_{o}, \lambda_{o}\right)$ and $\left(\phi_{1}, \lambda_{1}\right)$ be two distinct points in $J \times J \subset \mathbb{R}^{2}$. We want to find a curve $\gamma$ in $J \times J$ having the form $\gamma(t)=(\phi(t), \lambda(t))$ for all $t$ in I and a real number $\alpha$ in the interval $[0,2 \pi)$ such that:

- $\gamma(0)=\left(\phi_{o}, \lambda_{o}\right), \gamma(\epsilon)=\left(\phi_{1}, \lambda_{1}\right)$ for some positive scalar $\epsilon \in I$, and

- $\gamma^{\prime}(t)=\left[\frac{\cos \alpha}{r(\phi(t))} \frac{\sin \alpha}{R(\phi(t))}\right]^{T}$ for all $t$ in the closed interval $[0, \epsilon]$.

Proposition 5. Problem 1 has a solution if and only if Problem 2 has a solution. Moreover, the solution of Problem 1 is unique if and only if the solution of Problem 2 is unique. 
Proof: Suppose that Problem 1 is solvable and consider Problem 2. If $\eta$ is the solution curve for Problem 1 in the case when $p_{o}=\psi\left(\phi_{o}, \lambda_{o}\right)$ and $p_{1}=\psi\left(\phi_{1}, \lambda_{1}\right)$ then we claim that $\gamma:=\psi^{-1} \circ \eta$ is a solution for Problem 2. To see that, note that $\gamma(0)=\psi^{-1}\left(p_{o}\right)=\left(\phi_{o}, \lambda_{o}\right)$ and that $\gamma(\epsilon)=\psi^{-1}\left(p_{1}\right)=\left(\phi_{1}, \lambda_{1}\right)$. Moreover,

$$
\begin{aligned}
\gamma^{\prime}(t)=\left.\mathrm{d} \psi^{-1}\right|_{\eta(t)} \eta^{\prime}(t) & =\left.\mathrm{d} \psi^{-1}\right|_{\eta(t)}\left[(\cos \alpha) \mathbf{N}_{\mathbf{t}}+(\sin \alpha) \mathbf{E}_{\mathbf{t}}\right] \\
& =\left.(\cos \alpha) \mathrm{d} \psi^{-1}\right|_{\eta(t)} \mathbf{N}_{\mathbf{t}}+\left.(\sin \alpha) \mathrm{d} \psi^{-1}\right|_{\eta(t)} \mathbf{E}_{\mathbf{t}} \\
& =(\cos \alpha) \mathbf{n}_{\mathbf{t}}+(\sin \alpha) \mathbf{e}_{\mathbf{t}}=\left[\frac{\cos \alpha}{r(\phi(t))} \frac{\sin \alpha}{R(\phi(t))}\right]^{T}
\end{aligned}
$$

for all $t$ in the closed interval $[0, \epsilon]$. Hence, Problem 2 is solvable.

Conversely, if Problem 2 is solvable then we consider Problem 1. If $\gamma$ is the solution curve for Problem 2 in the case when $\left(\phi_{o}, \lambda_{o}\right)=\psi^{-1}\left(p_{o}\right)$ and $\left(\phi_{1}, \lambda_{1}\right)=$ $\psi^{-1}\left(p_{1}\right)$ then by a similar argument we can show that $\eta:=\psi \circ \gamma$ is a solution for Problem 1.

Moreover, since a solution of one problem is the image under a diffeomorphism of a solution of the other problem, it follows that a solution of one problem is unique if and only if a solution of the other problem is unique.

In the light of Proposition 5, in order to prove the existence of a unique solution for Problem 1, it is enough to prove the existence of a unique solution for Problem 2. Our strategy (which we follow in the proof of Theorem 8) is the following: show that there is a unique real number $\alpha$ in the interval $[0,2 \pi)$ for which there is a curve $\gamma$ that solves Problem 2 and that this curve is unique. We mention some preliminary results before we prove our main theorem, Theorem 8 .

The following lemma is a known result. Its proof and the relevant definitions can be found, e.g., in [1-3].

Lemma 6. Let $I$ be an open interval that contains $t_{o}$ and let $\Omega$ be an open bounded and connected subset of $\mathbb{R}^{n}$ that contains the point $\mathbf{u}_{\mathbf{o}}$. If $\mathbf{f}: I \times \Omega \rightarrow \mathbb{R}^{n}$ is a locally Lipschitz continuous vector-valued function (which holds, in particular, when $\mathbf{f}$ is a smooth function), then the initial value problem:

- $\mathbf{u}\left(t_{o}\right)=\mathbf{u}_{\mathbf{o}}$

- $\mathbf{u}^{\prime}(t)=\mathbf{f}(t, \mathbf{u}(t))$ for all $t$ in an open subinterval of I that contains $t_{o}$

has a unique solution that can be extended to the boundary of $\Omega$. 
Lemma 7. Let $\phi_{o}$ be a fixed real number in $J$ and let $\alpha$ be a real number in the interval $[0,2 \pi)$. There is a parametrization of the latitude variable $\phi(t)$ defined for $t$ in some open interval I that contains 0 such that $\phi(0)=\phi_{o}, \phi^{\prime}(t)=\frac{\cos \alpha}{r(\phi(t))}$ for all $t$ in $I$, and $\phi(t)$ assumes all values in $J$.

Proof: Let $\lambda_{o}$ be any real number in $J$ and consider the following initial value problem:

- $\gamma(0)=(\phi(0), \lambda(0))=\left(\phi_{o}, \lambda_{o}\right)$

- $\gamma^{\prime}(t)=\left[\begin{array}{ll}\phi^{\prime}(t) & \lambda^{\prime}(t)\end{array}\right]^{T}=\left[\begin{array}{ll}\frac{\cos \alpha}{r(\phi(t))} & 0\end{array}\right]^{T}$ for all $t$ in $I$.

Since the function $f(t, \phi, \lambda)=\left[\frac{\cos \alpha}{r(\phi)} 0\right]^{T}$ is a smooth function for all choices of $\alpha$ in the interval $[0,2 \pi)$, it follows by Lemma 6 that there is a unique curve $\gamma$ that extends to the boundary of $J \times J$ and that has the form $\gamma(t)=\left(\phi(t), \lambda_{o}\right)$ for all $t$ in $I$. The first entry of $\gamma(t)$, namely $\phi(t)$, is the desired parametrization of the latitude variable.

In the following theorem, the symbol $[g(\phi)]_{\phi=\phi_{o}}^{\phi=\phi_{1}}$ denotes $g\left(\phi_{1}\right)-g\left(\phi_{o}\right)$ if $g$ is a real-valued function of the latitude variable $\phi$.

Theorem 8. There is a unique $\alpha$ in the interval $[0,2 \pi)$ for which there is a curve $\gamma$ that solves Problem 2. Moreover, this curve $\gamma$ is unique. Furthermore, if $\phi_{o} \neq \phi_{1}$ then

$$
\tan \alpha=\frac{\lambda_{1}-\lambda_{o}}{\left[\ln \left(\left(\tan \left(\frac{\pi}{4}+\frac{\phi}{2}\right)\right)\left(\frac{1-e \sin \phi}{1+e \sin \phi}\right)^{\frac{e}{2}}\right)\right]_{\phi=\phi_{o}}^{\phi=\phi_{1}}}
$$

otherwise $\alpha=\frac{\pi}{2}$ (in the case when $\lambda_{1}>\lambda_{o}$ ) or $\frac{3 \pi}{2}$ (in the case when $\lambda_{1}<\lambda_{o}$ ).

Proof: Let us begin by considering the case when $\phi_{1}=\phi_{o}$. Since $\left(\phi_{o}, \lambda_{o}\right)$ and $\left(\phi_{1}, \lambda_{1}\right)$ are two distinct points in $J \times J$, we must have one of two subcases: i) $\lambda_{1}>\lambda_{o}$ or ii) $\lambda_{1}<\lambda_{o}$. Consider subcase i). By choosing $\alpha=\frac{\pi}{2}$ and $\epsilon=R\left(\phi_{o}\right)\left(\lambda_{1}-\lambda_{o}\right)$, it is straightforward to show that the curve $\gamma$ defined by $\gamma(t)=(\phi(t), \lambda(t)):=\left(\phi_{o}, \lambda_{o}+\frac{1}{R\left(\phi_{o}\right)} t\right)$ for all $t$ in the interval $[0, \epsilon]$ is a solution for Problem 2 in subcase i). Moreover, we claim that $\frac{\pi}{2}$ is the unique value of $\alpha$ in the interval $[0,2 \pi)$ for which there is a solution for Problem 2 in subcase i). Suppose with the hope of getting a contradiction that this is not true. Then, there is a real number $\beta_{o} \neq \frac{\pi}{2}$ in the interval $[0,2 \pi)$ such that Problem 2 has a solution in subcase i) if we substitute $\beta_{o}$ for $\alpha$. Note that $\beta_{o}$ can not be equal to $\frac{3 \pi}{2}$ otherwise 
$\phi(t)=\phi_{o}$ for all $t$ in $I$ (because $\phi^{\prime}(t)=\frac{\cos \beta_{o}}{r(\phi(t))}=0$ for all $t$ in $I$ ) and $\lambda(t)$ would be a decreasing function of $t$ (owing to the fact that $\lambda^{\prime}(t)=\frac{\sin \beta_{o}}{R(\phi(t))}=\frac{-1}{R\left(\phi_{o}\right)}<0$ ). Thus, the function $t \rightarrow \lambda(t)$ can not satisfy $\lambda(\epsilon)=\lambda_{1}>\lambda_{o}=\lambda(0)$ for a positive scalar $\epsilon$. Hence, $\beta_{o}$ is neither $\frac{\pi}{2}$ nor $\frac{3 \pi}{2}$, and as a result, $\cos \beta_{O}$ is either positive or negative. If $\cos \beta_{o}$ is positive, then, the function $\phi^{\prime}(t)=\frac{\cos \beta_{o}}{r(\phi(t))}>0$ for all $t$ in $I$ in spite that $0=\phi_{1}-\phi_{o}=\phi(\epsilon)-\phi(0)=\int_{0}^{\epsilon} \phi^{\prime}(t) \mathrm{d} t$ where $\epsilon$ is a positive scalar, a contradiction. Similarly, if $\cos \beta_{o}$ is negative we get a contradiction. Therefore, $\frac{\pi}{2}$ is the unique value of $\alpha$ in the interval $[0,2 \pi)$ for which Problem 2 in subcase i) has a solution. Moreover, this solution is unique and its uniqueness follows from Lemma 6. Similarly, one can show that $\frac{3 \pi}{2}$ is the unique value of $\alpha$ in the interval $[0,2 \pi)$ for which Problem 2 in subcase ii) has a solution and that this solution is unique.

Consider now the case when $\phi_{1} \neq \phi_{o}$. Then, we have one of two subcases: 1) $\phi_{1}>\phi_{o}$ or 2) $\phi_{1}<\phi_{o}$. Suppose that subcase 1) is true. Then, choose $\alpha$ in the interval $[0,2 \pi)$ so that:

$$
\tan \alpha=\left(\lambda_{1}-\lambda_{o}\right)\left(\int_{\phi_{o}}^{\phi_{1}} \frac{r(\phi)}{R(\phi)} \mathrm{d} \phi\right)^{-1} \quad \text { and } \quad \cos \alpha>0 .
$$

By Lemma 6, there is a unique curve $\phi \rightarrow \lambda(\phi)$ defined for all $\phi$ in $J$ and which solves the following initial value problem:

- $\lambda\left(\phi_{o}\right)=\lambda_{o}$

- $\frac{\mathrm{d} \lambda}{\mathrm{d} \phi}=\frac{r(\phi)}{R(\phi)} \tan \alpha$ for all $\phi$ in $J$.

This curve is in effect a parametrization of the longitude variable by the latitude variable. Moreover, let $\phi(t)$ be the parametrization of the latitude variable guaranteed by Lemma 7 . We claim that the curve $\gamma$ defined by $\gamma(t):=(\phi(t), \lambda(\phi(t)))$ is the unique curve that solves Problem 2 in subcase 1$)$. To see that, observe that $\gamma(0)=(\phi(0), \lambda(\phi(0)))=\left(\phi_{o}, \lambda\left(\phi_{o}\right)\right)=\left(\phi_{o}, \lambda_{o}\right)$. Moreover, since $\phi^{\prime}(t)=\frac{\cos \alpha}{r(\phi(t))}>0$, it follows that $\phi(t)$ is an increasing function of $t$ and by Lemma 7 this function assumes all values in $J$. Thus, for a sufficiently large positive scalar $\epsilon$ we have $\phi(\epsilon)=\phi_{1}$. And therefore,

$$
\begin{aligned}
\lambda(\phi(\epsilon))=\lambda\left(\phi_{1}\right) & =\int_{\phi_{o}}^{\phi_{1}} \frac{\mathrm{d} \lambda}{\mathrm{d} \phi} \mathrm{d} \phi+\lambda\left(\phi_{o}\right)=\int_{\phi_{o}}^{\phi_{1}} \frac{r(\phi)}{R(\phi)} \tan \alpha \mathrm{d} \phi+\lambda_{o} \\
& =\tan \alpha \int_{\phi_{o}}^{\phi_{1}} \frac{r(\phi)}{R(\phi)} \mathrm{d} \phi+\lambda_{o}=\left(\lambda_{1}-\lambda_{o}\right)+\lambda_{o}=\lambda_{1} .
\end{aligned}
$$


Hence, $\gamma(\epsilon)=\left(\phi_{1}, \lambda_{1}\right)$. Furthermore,

$$
\begin{aligned}
\gamma^{\prime}(t)=\left[\phi^{\prime}(t) \frac{\mathrm{d} \lambda}{\mathrm{d} \phi} \frac{\mathrm{d} \phi}{\mathrm{d} t}\right]^{T} & =\left[\frac{\cos \alpha}{r(\phi(t))}\left(\frac{r(\phi(t))}{R(\phi(t))} \tan \alpha\right)\left(\frac{\cos \alpha}{r(\phi(t))}\right)\right]^{T} \\
& =\left[\frac{\cos \alpha}{r(\phi(t))} \frac{\sin \alpha}{R(\phi(t))}\right]^{T} .
\end{aligned}
$$

Thus, for the particular choice of $\alpha$ specified in (6) we showed that the curve $\gamma$ is the unique solution for Problem 2 in subcase 1). Moreover, there is no other value of $\alpha$ for which Problem 2 in subcase 1) has a solution. To see that, suppose to the contrary that there is an $\tilde{\alpha}$ in the interval $[0,2 \pi)$ and a curve $\gamma$ defined on an interval $I$ that contains 0 such that:

- $\tilde{\alpha}$ is not equal to the value of $\alpha$ given in (6),

- $\gamma(0)=\left(\phi_{o}, \lambda_{o}\right), \gamma(\epsilon)=\left(\phi_{1}, \lambda_{1}\right)$ for some positive scalar $\epsilon$ in $I$, and

- $\gamma^{\prime}(t)=\left[\begin{array}{ll}\phi^{\prime}(t) & \lambda^{\prime}(t)\end{array}\right]^{T}=\left[\begin{array}{ll}\frac{\cos \tilde{\alpha}}{r(\phi(t))} & \frac{\sin \tilde{\alpha}}{R(\phi(t))}\end{array}\right]^{T}$ for all $t$ in the closed interval $[0, \epsilon]$.

Since $\phi_{1}>\phi_{o}$ and since $\phi^{\prime}(t)=\frac{\cos \tilde{\alpha}}{r(\phi(t))}$, we must have $\cos \tilde{\alpha}>0$ otherwise $\phi(t)$ would be a decreasing function of $t$. Moreover, note that $\frac{\lambda^{\prime}(t)}{\phi^{\prime}(t)}=\frac{r(\phi(t))}{R(\phi(t))} \tan \tilde{\alpha}$, and thus, $\lambda^{\prime}(t)=\frac{r(\phi(t))}{R(\phi(t))} \phi^{\prime}(t) \tan \tilde{\alpha}$. Hence,

$$
\begin{aligned}
\lambda_{1}-\lambda_{o}=\lambda(\epsilon)-\lambda(0) & =\int_{0}^{\epsilon} \lambda^{\prime}(t) \mathrm{d} t=\int_{0}^{\epsilon} \frac{r(\phi(t))}{R(\phi(t))} \phi^{\prime}(t) \tan \tilde{\alpha} \mathrm{d} t \\
& =\tan \tilde{\alpha} \int_{0}^{\epsilon} \frac{r(\phi(t))}{R(\phi(t))} \phi^{\prime}(t) \mathrm{d} t=\tan \tilde{\alpha} \int_{\phi_{o}}^{\phi_{1}} \frac{r(\phi)}{R(\phi)} \mathrm{d} \phi .
\end{aligned}
$$

And therefore,

$$
\tan \tilde{\alpha}=\left(\lambda_{1}-\lambda_{o}\right)\left(\int_{\phi_{o}}^{\phi_{1}} \frac{r(\phi)}{R(\phi)} \mathrm{d} \phi\right)^{-1} .
$$

But this implies that $\tilde{\alpha}$ is equal to the value of $\alpha$ given in (6), a contradiction. Thus, there is a unique value of $\alpha$ in the interval [0,2 $2 \pi$ ) given by (6) for which Problem 2 in subcase 1) has a solution. Moreover, this solution is unique by Lemma 6 .

If subcase 2) holds then by a similar argument one can show that if we choose $\alpha$ in the interval $[0,2 \pi)$ so that:

$$
\tan \alpha=\left(\lambda_{1}-\lambda_{o}\right)\left(\int_{\phi_{o}}^{\phi_{1}} \frac{r(\phi)}{R(\phi)} \mathrm{d} \phi\right)^{-1} \quad \text { and } \quad \cos \alpha<0
$$


then Problem 2 in subcase 2) has a solution and the value of $\alpha$ given in (7) is the only value of $\alpha$ in the interval $[0,2 \pi)$ such that Problem 2 in subcase 2) has a solution. Moreover, this solution is unique by Lemma 6 .

We finally note that the form of $\tan \alpha$ given in (6) and (7) is the same as that given in (5). To see that, observe that

$$
\begin{aligned}
\int_{\phi_{o}}^{\phi_{1}} \frac{r(\phi)}{R(\phi)} \mathrm{d} \phi= & \int_{\phi_{o}}^{\phi_{1}} \frac{\left(1-e^{2}\right)}{\left(1-e^{2} \sin ^{2} \phi\right) \cos \phi} \mathrm{d} \phi \\
= & \int_{\phi_{o}}^{\phi_{1}} \frac{1}{\cos \phi} \mathrm{d} \phi+\int_{\phi_{o}}^{\phi_{1}} \frac{-\frac{e^{2}}{2} \cos \phi}{1-e \sin \phi} \mathrm{d} \phi+\int_{\phi_{o}}^{\phi_{1}} \frac{-\frac{e^{2}}{2} \cos \phi}{1+e \sin \phi} \mathrm{d} \phi \\
= & {\left[\ln \left(\tan \left(\frac{\pi}{4}+\frac{\phi}{2}\right)\right)\right]_{\phi=\phi_{o}}^{\phi=\phi_{1}}+\left[\frac{e}{2} \ln (1-e \sin \phi)\right]_{\phi=\phi_{o}}^{\phi=\phi_{1}} } \\
& +\left[-\frac{e}{2} \ln (1+e \sin \phi)\right]_{\phi=\phi_{o}}^{\phi=\phi_{1}} \\
= & {\left.\left[\ln \left(\tan \left(\frac{\pi}{4}+\frac{\phi}{2}\right)\right)\left(\frac{1-e \sin \phi}{1+e \sin \phi}\right)^{\frac{e}{2}}\right)\right]_{\phi=\phi_{o}}^{\phi=\phi_{1}} . }
\end{aligned}
$$

Hence, the form of $\tan \alpha$ given in (6) and (7) reduces to the form given in (5).

Corollary 9. There is a unique $\alpha$ in the interval $[0,2 \pi)$ for which there is a curve $\eta$ that solves Problem 1. Moreover, this curve $\eta$ is unique. Furthermore, if $\psi^{-1}\left(p_{o}\right)=\left(\phi_{o}, \lambda_{o}\right)$ and $\psi^{-1}\left(p_{1}\right)=\left(\phi_{1}, \lambda_{1}\right)$, then $\alpha$ is as described in Theorem 8, i.e., if $\phi_{o} \neq \phi_{1}$ then $\tan \alpha$ is given by (5) otherwise $\alpha=\frac{\pi}{2}$ (when $\lambda_{1}>\lambda_{o}$ ) or $\alpha=\frac{3 \pi}{2}\left(\right.$ when $\left.\lambda_{1}<\lambda_{o}\right)$.

\section{Appendix}

We present here the postponed proofs of some of the results

Proof of Lemma 2: Let us first prove that the first entry (respectively, the second entry) of $\psi^{-1}(x, y, z)$ is the latitude (respectively, the longitude) of the point $(x, y, z)$. Let $p=(x, y, z)$ be any point in $\mathcal{E}^{+}$and let $\phi$ and $\lambda$ be its corresponding latitude and longitude, respectively. Since $\sin \lambda=\frac{y}{\sqrt{x^{2}+y^{2}}}$ (see Fig. 2), it follows that $\lambda=\arcsin \left(\frac{y}{\sqrt{x^{2}+y^{2}}}\right)$, i.e., the longitude of $p=(x, y, z)$ is the second entry of $\psi^{-1}(x, y, z)$. 

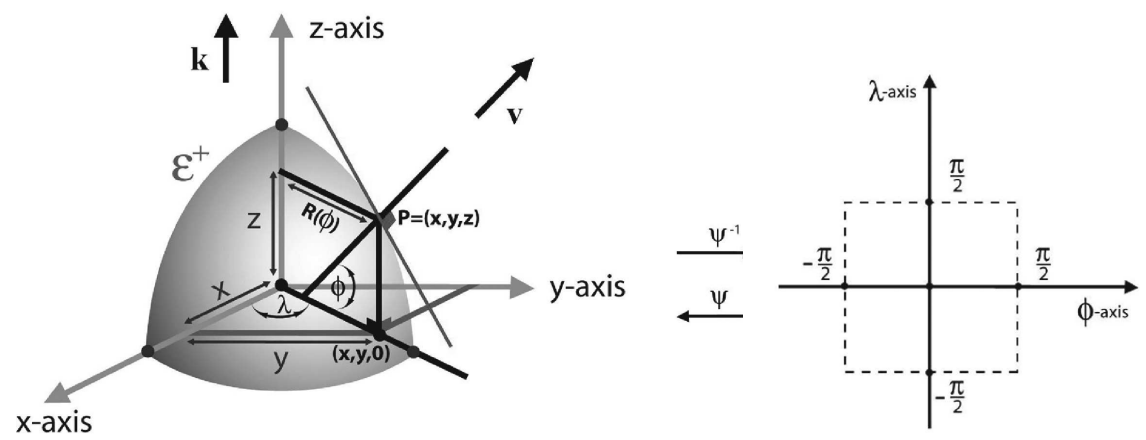

Figure 2. The diffeomorphism $\psi$ and its inverse.

Moreover, the angle between the normal line to $\mathcal{E}^{+}$at $p$ and the $z$-axis is $\frac{\pi}{2}-$ $\phi$; see Fig. 2. If $\mathbf{v}$ denotes the outward unit normal vector at $p$ and $\mathbf{k}$ denotes the unit vector pointing in the direction of increasing values on the $z$-axis, then by taking the inner product of $\mathbf{v}$ with $\mathbf{k}$ one can find $\cos \left(\frac{\pi}{2}-\phi\right)$. Indeed, if we let $\|\cdot\|$ denote the Euclidean vector norm, let $\langle$,$\rangle denote the Euclidean$ inner product, and let $\mathbf{w}=\left[\begin{array}{lll}\frac{2 x}{a^{2}} & \frac{2 y}{a^{2}} & \frac{2 z}{b^{2}}\end{array}\right]^{T}$, then we have the following. The vector $\mathbf{v}=\frac{1}{\|\mathbf{w}\|} \mathbf{w}$, the vector $\mathbf{k}=\left[\begin{array}{lll}0 & 0 & 1\end{array}\right]^{T}$, and $\sin \phi=\cos \left(\frac{\pi}{2}-\phi\right)=$ $\langle\mathbf{k}, \mathbf{v}\rangle=\frac{z / b^{2}}{\sqrt{\left(x^{2}+y^{2}\right) / a^{4}+z^{2} / b^{4}}} \cdot$ Since $\frac{x^{2}+y^{2}}{a^{2}}+\frac{z^{2}}{b^{2}}=1$ and since $|\phi|<\frac{\pi}{2}$ ( $\phi$ being the latitude of a point $p=(x, y, z)$ in $\mathcal{E}^{+}$), it follows that $\cos \phi=$ $\frac{\left(x^{2}+y^{2}\right)^{1 / 2} / a^{2}}{\sqrt{\left(x^{2}+y^{2}\right) / a^{4}+z^{2} / b^{4}}}$, and hence,

$$
\tan \phi=\frac{a^{2} z}{b^{2}\left(x^{2}+y^{2}\right)^{1 / 2}}=\frac{z}{\left(1-e^{2}\right)\left(x^{2}+y^{2}\right)^{1 / 2}} .
$$

Therefore,

$$
\phi=\arctan \left(\frac{z}{\left(1-e^{2}\right) \sqrt{x^{2}+y^{2}}}\right)
$$

i.e., the longitude of $p=(x, y, z)$ is the first entry of $\psi^{-1}(x, y, z)$. Furthermore, 
we note that since $\tan \phi$ is given by ( 8 ) and since $\frac{x^{2}+y^{2}}{a^{2}}+\frac{z^{2}}{b^{2}}=1$, it follows that

$$
\begin{aligned}
R(\phi) & =a \cos \phi\left(1-e^{2} \sin ^{2} \phi\right)^{-1 / 2}=a\left(1+\left(1-e^{2}\right) \tan ^{2} \phi\right)^{-1 / 2} \\
& =a\left(1+\frac{a^{2} z^{2}}{b^{2}\left(x^{2}+y^{2}\right)}\right)^{-1 / 2}=\sqrt{x^{2}+y^{2}} .
\end{aligned}
$$

And thus, it becomes straightforward (with the aid of Fig. 2) to verify that $\psi \circ \psi^{-1}=$ Id and $\psi^{-1} \circ \psi=$ Id for the expressions for $\psi$ and $\psi^{-1}$ given in equations (1) and (3). Moreover, the smoothness of $\psi$ and $\psi^{-1}$ follow from the following facts about smooth functions of one variable: i) linear combinations, products, and compositions of smooth functions are smooth functions, ii) if $f$ is a smooth function then so is $\frac{1}{f}$ on the set $\{x \in$ Domain of $f ; f(x) \neq 0\}$, and iii) if $f$ is a smooth function then so is $\sqrt{f}$ on the set $\{x \in$ Domain of $f ; f(x)>0\}$.

Proof of Lemma 3: It is clear that the Euclidean norm of the vector $\mathbf{N}_{\mathbf{t}}$ (respectively, $\mathbf{E}_{\mathbf{t}}$ ) is 1 . Moreover, since $\psi$ is a diffeomorphism and since $(\phi(t), \lambda(t))=\gamma(t)=\psi \circ \eta(t)$, it follows that $\phi(t)$ and $\lambda(t)$ depend smoothly on the parameter $t$, and thus, the vector $\mathbf{N}_{\mathbf{t}}$ (respectively, $\mathbf{E}_{\mathbf{t}}$ ) depends smoothly on the parameter $t$. Note that the vector $\mathbf{N}_{\mathbf{t}}$ (respectively, $\mathbf{E}_{\mathbf{t}}$ ) points towards the north (respectively, the east) since its $z$-component (respectively, $y$-component) is positive.

Furthermore, differentiating $R(\phi)$ with respect to $\phi$, one gets:

$$
\begin{aligned}
\frac{\mathrm{d} R}{\mathrm{~d} \phi} & =(-a \sin \phi)\left(1-e^{2} \sin \phi\right)^{-1 / 2}+a e^{2} \cos \phi \sin \phi\left(1-e^{2} \sin \phi\right)^{-3 / 2} \\
& =a \sin \phi\left(1-e^{2} \sin \phi\right)^{-3 / 2}\left[-\left(1-e^{2} \sin ^{2} \phi\right)+e^{2} \cos \phi\right] \\
& =a \sin \phi\left(1-e^{2} \sin \phi\right)^{-3 / 2}\left[-1+e^{2}\right]=-r(\phi) .
\end{aligned}
$$

And thus,

$$
\begin{aligned}
& \mathrm{d} \psi=\left[\begin{array}{cc}
-r(\phi) \sin \phi \cos \lambda & -R(\phi) \sin \lambda \\
-r(\phi) \sin \phi \sin \lambda & -R(\phi) \cos \lambda \\
r(\phi) \cos \phi & 0
\end{array}\right] \\
& \mathrm{d} \psi\left[0 \frac{1}{R(\phi)}\right]^{T}=\left[\begin{array}{lll}
-\sin \lambda & \cos \lambda & 0
\end{array}\right]^{T}
\end{aligned}
$$

and $\mathrm{d} \psi\left[\begin{array}{ll}\frac{1}{r(\phi)} & 0\end{array}\right]^{T}=\left[\begin{array}{lll}-\sin \phi \cos \lambda-\sin \phi \sin \lambda & \cos \phi\end{array}\right]^{T}$. In particular, when $\phi$ and $\lambda$ are parameterized by a real variable $t$, the abovementioned matrix equalities hold. Hence, $\left.\mathrm{d} \psi\right|_{\gamma(t)} \mathbf{e}_{\mathbf{t}}=\mathbf{E}_{\mathbf{t}}$ and $\left.\mathrm{d} \psi\right|_{\gamma(t)} \mathbf{n}_{\mathbf{t}}=\mathbf{N}_{\mathbf{t}}$. Since $\left.\left.\mathrm{d} \psi^{-1}\right|_{\eta(t)} \circ \mathrm{d} \psi\right|_{\gamma(t)}$ $=\operatorname{Id}_{\mathbb{R}^{2}}$, it follows that the claim of this lemma about the form of $\mathbf{n}_{\mathbf{t}}$ and $\mathbf{e}_{\mathbf{t}}$ is true. 


\title{
References
}

[1] Bourbaki N., Elements of Mathematics, Springer, New York, 2004.

[2] Coddington E. and Levinson N., Theory of Ordinary Differential Equations, McGraw-Hill, New York, 1955.

[3] Cole R., Theory of Ordinary Differential Equations, Appleton Century Crofts, New York, 1968.

[4] Hofmann-Wellenhof B., Legat K., Wieser M. and Lichtenegger H., Navigation: Principles of Positioning and Guidance, Springer, New York, 2003.

[5] Pearson F., Map Projections: Theory and Applications, CRC Press, Boca Raton, 1990.

[6] Smart W., Text-Book on Spherical Astronomy, Cambridge University Press, Cambridge, 1947.

[7] Spivak M., A Comprehensive Introduction to Differential Geometry, vol. 1, Publish or Perish Inc., Willmington, 1979.

[8] Warner F., Foundations of Differentiable Manifolds and Lie Groups, Springer, New York, 1983.

[9] Williams R., Geometry of Navigation, Horwood Publ., West Sussex, 1998.

\author{
Abed Elhashash \\ Department of Mathematics \\ Drexel University \\ 3141 Chestnut Street \\ Philadelphia, PA 19104 \\ USA \\ E-mail address: aae36@drexel .edu
}

